\title{
The Blocking Numbers of Convex Bodies*
}

\author{
L. Dalla, ${ }^{1}$ D. G. Larman, ${ }^{2}$ P. Mani-Levitska, ${ }^{3}$ and C. Zong $^{4}$ \\ ${ }^{1}$ Department of Mathematics, University of Athens, \\ Panepistemiopolis, 15481 Athens, Greece \\ 2 Department of Mathematics, University College London, \\ Gower Street, London WC1E 6BT, England \\ dgl@math.ucl.ac.uk \\ ${ }^{3}$ Mathematisches Institut, Universität Bern, \\ Sidlerstrasse 5, CH-3012 Bern, Switzerland \\ ${ }^{4}$ Institute of Mathematics, The Chinese Academy of Sciences, \\ Beijing 100080, People's Republic of China \\ cmzong@math08.math.ac.cn
}

\begin{abstract}
Besides determining the exact blocking numbers of cubes and balls, a conditional lower bound for the blocking numbers of convex bodies is achieved. In addition, several open problems are proposed.
\end{abstract}

\section{Introduction}

Let $K$ be a convex body in $R^{n}$. The blocking number $b(K)$ of $K$ is the smallest number of nonoverlapping translates $K+\mathbf{x}$ which are in contact with $K$ at its boundary and prevent any other translate from touching it. Similarly, we can define a number $b^{\prime}(K)$ by allowing overlapping between the translates.

As a counterpart and a limited case of kissing numbers, blocking numbers were first introduced in [12] and studied in [13] and [14], where an inequality between blocking numbers and Hadwiger's covering numbers for centrally symmetric convex bodies and results concerning blocking numbers and kissing numbers have been achieved. Nevertheless, so far our knowledge about blocking numbers is very limited.

\footnotetext{
* This work was done while the first and the last authors were guests of the Mathematical Department of the University College London. The work of the last author was supported by a research fellowship of The Royal Society.
} 


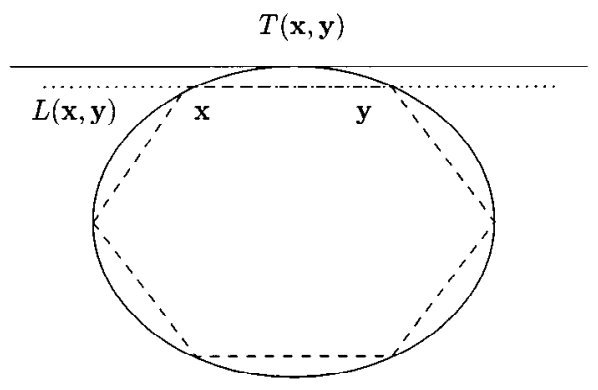

Fig. 1

Denote the difference set of a convex set $X$ by $D(X)$. In other words, $D(X)=$ $\{\mathbf{x}-\mathbf{y}: \mathbf{x}, \mathbf{y} \in X\}$. It is well known that $\left(X+\mathbf{x}_{1}\right) \cap\left(X+\mathbf{x}_{2}\right) \neq \emptyset$ if and only if $\left(\frac{1}{2} D(X)+\mathbf{x}_{1}\right) \cap\left(\frac{1}{2} D(X)+\mathbf{x}_{2}\right) \neq \varnothing$ (see [5]). Since $D(K)$ is centrally symmetric, we may confine ourselves to the centrally symmetric case whenever we deal with the kissing numbers and the blocking numbers (this is not true for Hadwiger's covering numbers) of convex bodies. In addition, we may apply the Minkowski metric $\|\cdot\|_{C}$ given by a centrally symmetric convex body $C$ to our problem (for the definition of $\|\cdot\|_{C}$ we refer to [9]).

Definition 1. Let $C$ be a centrally symmetric convex body in $R^{n}$, let $\partial(C)$ be its boundary, and let $\|\cdot\|_{C}$ be the Minkowski metric given by $C$. Denote the manifold $\left\{[\mathbf{x}, \mathbf{y}]: \mathbf{x}, \mathbf{y} \in \partial(C) ;\|\mathbf{x}, \mathbf{y}\|_{C}=1\right\}$ by $\Phi$, the straight line passing $\mathbf{x}$ and $\mathbf{y}$ by $L(\mathbf{x}, \mathbf{y})$, the two-dimensional plane passing $\mathbf{o}, \mathbf{x}$, and $\mathbf{y}$ by $P(\mathbf{x}, \mathbf{y})$, and the tangent of $C \cap P(\mathbf{x}, \mathbf{y})$ which is parallel to $L(\mathbf{x}, \mathbf{y})$ and is at the same side of $\mathbf{o}$ with $L(\mathbf{x}, \mathbf{y})$ by $T(\mathbf{x}, \mathbf{y})$ (see Fig. 1). Then we call

$$
m(C)=\min _{[\mathbf{x}, \mathbf{y}] \in \Phi}\left\{1-\frac{d(\mathbf{o}, L(\mathbf{x}, \mathbf{y}))}{d(\mathbf{o}, T(\mathbf{x}, \mathbf{y}))}\right\}
$$

the $M$-curvature of $C$, where $d(X, Y)$ indicates the Euclidean distance between the two sets $X$ and $Y$.

Clearly, the $M$-curvature of a centrally symmetric convex body is invariant when a linear transformation is applied to it. In high dimensions we have the following bounds for $m(C)$.

Theorem 1. For any positive number $\varepsilon$ there is an integer $N(\varepsilon)$ such that

$$
0 \leq m(C) \leq 1+\varepsilon-\frac{\sqrt{3}}{2}
$$

holds for every $n$-dimensional centrally symmetric convex body $C$ whenever $n \geq N(\varepsilon)$.

This theorem is an immediate consequence of Dvoretzky's well-known theorem (see [3] or [14]) which asserts: Let $B$ be the $n$-dimensional unit ball, and let $\varepsilon$ be a positive number. For each $k$-dimensional centrally symmetric convex body $C$, where $k$ is an 
integer determined by $n$ and $\varepsilon$, there exist an $n$-dimensional subspace $R^{n}$ of $R^{k}$ and $a$ positive number $r$ such that

$$
r B \subseteq C \cap R^{n} \subseteq(1+\varepsilon) r B .
$$

Problem 1. Determine the value $\max \{m(C)\}$, where the maximum is over all centrally symmetric convex domains. We conjecture that the regular 10-gon is the extremal figure.

Problem 2. Is it true that $b(K)=b^{\prime}(K)$ for all convex bodies?

\section{A Lower Bound of $b(C)$ Based on the $M$-Curvature of $C$}

Lemma 1 [8] (see also [1]). For every $n$-dimensional centrally symmetric convex body $C$ there exists a linear transformation $T$ such that

$$
B \subseteq T(C) \subseteq \sqrt{n} B
$$

By this lemma, since both the M-curvature and the blocking number of a centrally symmetric convex body are invariant when a linear transformation is applied to it, we only deal with the $n$-dimensional centrally symmetric convex bodies which satisfy (1). As usual, let $\partial(K)$ be the boundary of a convex body $K$, and let $v_{m}(X)$ be the $m$-dimensional lebesgue measure of a set $X$. In addition, we denote by $S_{\mathbf{u}}$ the Steiner symmetrization with respect to the hyperplane with norm $\mathbf{u}$.

Lemma 2 [11]. For every $n$-dimensional convex body $K$ and every direction $\mathbf{u}$,

$$
v_{n-1}(\partial(K)) \geq v_{n-1}\left(\partial\left(S_{\mathbf{u}}(K)\right)\right) .
$$

Lemma 3 (see [7]). Let $C$ be a centrally symmetric convex domain in $R^{2}$. For every point $\mathbf{x} \in \partial(C)$ there exists an affine regular hexagon inscribed in $C$ which takes $\mathbf{x}$ as one of its six vertices.

Theorem 2. If $C$ is an n-dimensional centrally symmetric convex body with M-curvature $m(C)$, then

$$
b(C) \geq n^{-3 / 2}(1-m(C))^{2-n} .
$$

Proof. We assume that $n \geq 3$. As usual, denote the interior of $C$ by $\operatorname{int}(C)$. Assume that $X=\left\{\mathbf{x}_{0}=\mathbf{o}, \mathbf{x}_{1}, \mathbf{x}_{2}, \ldots, \mathbf{x}_{b(C)}\right\}$ is a set of points such that $\left(\operatorname{int}(C)+\mathbf{x}_{i}\right) \cap\left(\operatorname{int}(C)+\mathbf{x}_{j}\right)=$ $\emptyset$ whenever $i \neq j, C+\mathbf{x}_{i}$ touches $C$ at its boundary for every point $\mathbf{x}_{i} \in X$, and $\bigcup_{i=1}^{b(C)}\left(C+\mathbf{x}_{i}\right)$ prevents any other translate of $C$ from touching it. For convenience, in the following discussion we call $X \backslash\{\mathbf{o}\}$ a blocking set of $C$. Then, observing the boundary of $2 C$, one obtains

$$
\partial(2 C) \subset \bigcup_{i=1}^{b(C)}\left(\operatorname{int}(2 C)+\mathbf{x}_{i}\right)
$$


and hence

$$
\sum_{i=1}^{b(C)} \frac{v_{n-1}\left(\partial(2 C) \cap\left(\operatorname{int}(2 C)+\mathbf{x}_{i}\right)\right)}{v_{n-1}(\partial(2 C))} \geq 1 .
$$

Now, we proceed to show

$$
\frac{v_{n-1}\left(\partial(2 C) \cap\left(\operatorname{int}(2 C)+\mathbf{x}_{i}\right)\right)}{v_{n-1}(\partial(2 C))} \leq n^{3 / 2}(1-m(C))^{n-2}
$$

for all indices $i=1,2, \ldots, b(C)$.

Let $P_{i}$ be the perpendicular projection from $R^{n}$ to the hyperplane which contains $\mathbf{o}$ and takes $\mathbf{x}_{i}$ as a norm. Keeping (1) in mind, we have

$$
\operatorname{conv}\left\{-\mathbf{x}_{i}^{*}, P_{i}(2 C), \mathbf{x}_{i}^{*}\right\} \subseteq S_{\mathbf{x}_{i}^{*}}(2 C),
$$

where $\mathbf{x}_{i}^{*}$ indicates the unit direction of $\mathbf{x}_{i}$ and $\operatorname{conv}\{X\}$ indicates the convex hull of a set $X$.

Abbreviating $v_{n-2}\left(\partial\left(P_{i}(2 C)\right)\right)$ to $\mu_{i}$, for every positive number $r$ we have

$$
v_{n-2}\left(\partial\left(r P_{i}(2 C)\right)\right)=\mu_{i} r^{n-2} .
$$

Thus,

$$
v_{n-1}\left(\partial\left(\operatorname{conv}\left\{-\mathbf{x}_{i}^{*}, P_{i}(2 C), \mathbf{x}_{i}^{*}\right\}\right)\right) \geq 2 \mu_{i} \int_{0}^{1}(1-t)^{n-2} d t=\frac{2 \mu_{i}}{n-1} .
$$

Therefore, by (4), (5), and Lemma 2 we obtain

$$
v_{n-1}(\partial(2 C)) \geq \frac{2 \mu_{i}}{n-1} .
$$

On the other hand, defining a cylinder

$$
H_{i}=\left\{(1-m(C)) P_{i}(2 C)+\theta \mathbf{x}_{i}^{*}:|\theta| \leq \sqrt{n}\right\},
$$

it is easy to calculate

$$
v_{n-1}\left(\partial\left(H_{i}\right)\right)=2 \mu_{i} \sqrt{n}(1-m(C))^{n-2}+\frac{2 \mu_{i}(1-m(C))^{n-1}}{n-1} .
$$

Meanwhile, by (1), Lemma 3, and the definition of $m(C)$ (see Fig. 2), for every index $i$ we have

$$
\operatorname{conv}\left\{\partial(2 C) \cap\left(\operatorname{int}(2 C)+\mathbf{x}_{i}\right), \partial(2 C) \cap\left(\operatorname{int}(2 C)-\mathbf{x}_{i}\right)\right\} \subseteq H_{i} .
$$

It is well known that

$$
v_{n-1}\left(\partial\left(K_{1}\right)\right) \leq v_{n-1}\left(\partial\left(K_{2}\right)\right)
$$

whenever $K_{1} \subseteq K_{2}$. So, (7) and (8) yield

$$
v_{n-1}\left(\partial(2 C) \cap\left(\operatorname{int}(2 C)+\mathbf{x}_{i}\right)\right) \leq \mu_{i} \sqrt{n}(1-m(C))^{n-2}+\frac{\mu_{i}(1-m(C))^{n-1}}{n-1} .
$$

Clearly, (3) is a consequence of (6) and (9). Theorem 2 follows from (2) and (3). 


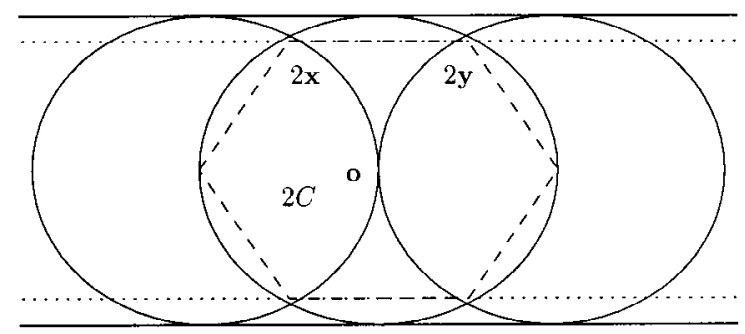

Fig. 2

If $\partial(C)$ is smooth, a similar (but worse) inequality between $b(C)$ and the minimal section curvature of $\partial(C)$ can be achieved by this method.

Concerning the blocking number of a convex body and the blocking numbers of its sections we have the following result.

Theorem 3. There are infinitely many dimensions in which a centrally symmetric convex body $C$ and a proper subspace $R$ can be found such that

$$
b(C) \leq b(C \cap R)+1 .
$$

Proof. If there exist only finite many such dimensions, we assume that $n-1$ is the last one. By Theorem 2, we may choose a positive number $\varepsilon$ such that

$$
b(D)>2 n
$$

holds for every $n$-dimensional centrally symmetric convex body $D$ which satisfies

$$
B \subseteq D \subseteq(1+\varepsilon) B .
$$

Let $k$ be the integer determined by $n$ and $\varepsilon$ in Dvoretzky's theorem. According to a result of [12], there is a $k$-dimensional centrally symmetric convex body $C$ such that

$$
b(C) \leq 2 k .
$$

Meanwhile, by Dvoretzky's theorem, $C$ has an $n$-dimensional section $D$ which satisfies (11) (note $b(r D)=b(D)$ ), and therefore (10). Choose a sequence of subspaces $R^{n} \subset$ $R^{n+1} \subset \cdots \subset R^{k}$ such that $D=C \cap R^{n}$ and $C \subset R^{k}$. According to our assumption,

$$
b\left(C \cap R^{n+i+1}\right) \geq b\left(C \cap R^{n+i}\right)+2
$$

holds for every index $i, 0 \leq i \leq k-n-1$. So that by (10) we obtain

$$
b(C)>2 n+2(k-n) \geq 2 k,
$$

a contradiction to (12). Theorem 3 is proved.

Problem 3. Is there an $m$-dimensional Euclidean space in which a centrally symmetric convex body $C$ and an $(m-1)$-dimensional subspace $R^{m-1}$ can be found such that

$$
b(C) \leq(\text { or even }<) b\left(C \cap R^{m-1}\right) ?
$$




\section{The Blocking Numbers of Some Special Convex Bodies}

In [12], based on a result of [6] about kissing numbers, it is proved that $b(K)=4$ for every two-dimensional convex domain. Now, we proceed to determine the blocking numbers of cubes (in general dimensions) and balls, respectively. In fact we can also prove that the blocking number of a tetrahedron is six.

\subsection{Cubes}

Theorem 4. The blocking number of an n-dimensional cube is $2^{n}$.

Proof. Denote the unit cube $\left\{\left(x^{1}, x^{2}, \ldots, x^{n}\right):\left|x^{i}\right| \leq \frac{1}{2}\right\}$ in $R^{n}$ by $W$, and let $X$ be a blocking set of $W$. Repeating partial argument in the proof of Theorem 2, one gets

$$
\partial(2 W) \subset \bigcup_{\mathbf{x} \in X}(\operatorname{int}(2 W)+\mathbf{x}) .
$$

Since each translate of $\operatorname{int}(2 W)$ contains at most one vertex of $2 W$, it follows immediately that

$$
b(W) \geq 2^{n}
$$

Now, we proceed to prove

$$
b(W) \leq 2^{n}
$$

by constructing a proper blocking set of $W$. If $n=2$, it can be verified that $\left\{\left(1, \frac{1}{2}\right),\left(\frac{1}{2}, 1\right)\right.$, $\left.\left(-1, \frac{1}{2}\right),\left(-\frac{1}{2},-1\right)\right\}$ is a blocking set of $W$. Inductively, if $X=\left\{\mathbf{x}_{1}, \mathbf{x}_{2}, \ldots, \mathbf{x}_{2^{n-1}}\right\}$ is a centrally symmetric blocking set of the $(n-1)$-dimensional unit cube and $\mathbf{x}_{1}=-\mathbf{x}_{2^{n-1}}$, we define

$$
\mathbf{y}_{i}= \begin{cases}\left(\frac{1}{3}, \frac{1}{3}, \ldots, \frac{1}{3}\right) \oplus 1, & i=1 \\ \mathbf{x}_{i} \oplus \frac{1}{2}, & i=2, \ldots, 2^{n-1} \\ \mathbf{x}_{i-2^{n-1} \oplus\left(-\frac{1}{2}\right),} & i=2^{n-1}+1, \ldots, 2^{n}-1 \\ \left(-\frac{1}{3},-\frac{1}{3}, \ldots,-\frac{1}{3}\right) \oplus(-1), & i=2^{n}\end{cases}
$$

where $\left(x^{1}, x^{2}, \ldots, x^{n-1}\right) \oplus x$ indicates $\left(x^{1}, x^{2}, \ldots, x^{n-1}, x\right)$ in $R^{n}$. Taking $Y=\left\{\mathbf{y}_{1}, \mathbf{y}_{2}\right.$, $\left.\ldots, \mathbf{y}_{2^{n}}\right\}$, it can be verified that $Y$ is a centrally symmetric blocking set of the $n$-dimensional unit cube. Hence we get (15). Theorem 4 follows from (14) and (15).

\subsection{Balls}

Theorem 5. The blocking number of a three-dimensional ball is six.

Proof. Denote the three-dimensional unit ball by $B$. Since $\{ \pm(2,0,0), \pm(0,2,0)$, $\pm(0,0,2)\}$ is a blocking set of $\mathrm{B}$, one gets

$$
b(B) \leq 6 .
$$




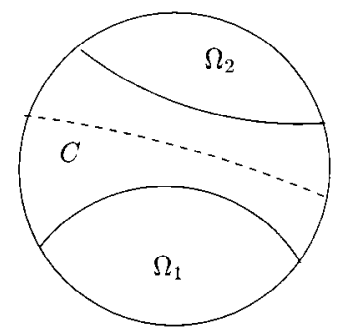

Fig. 3

On the other hand, assume that $X=\left\{\mathbf{x}_{1}, \mathbf{x}_{2}, \ldots, \mathbf{x}_{b(B)}\right\}$ is a blocking set of $B$, then

$$
\partial(2 B)=\bigcup_{i=1}^{b(B)} \Omega_{i},
$$

where $\Omega_{i}$ indicates the relative open cap $\partial(2 B) \cap\left(\operatorname{int}(2 B)+\mathbf{x}_{i}\right)$. Clearly, the angle between any two vectors which belong to the same cap is less than $2 \pi / 3$. So that any great circle of $\partial(2 B)$ intersects at least four of these $b(B)$ caps. If, without loss of generality, $-\mathbf{x}_{1} \in \Omega_{2}$, it can be shown that $\Omega_{1} \cap \Omega_{2}=\emptyset$. Then $\partial(2 B)$ has a great circle $C$ which intersects neither $\Omega_{1}$ nor $\Omega_{2}$. Therefore, we get $b(B) \geq 6$, which together with (16) prove our theorem.

Remark 1. This simple result can also be deduced from a result of Fejes Tóth [4] about cap covering density.

In 1993, at a geometric meeting held in Vienna, Zong proposed the following problem.

Problem 4. Denote by $\delta^{H}\left(K_{1}, K_{2}\right)$ the Hausdorff distance between $K_{1}$ and $K_{2}$ (see [9] for the definition). Let $B$ be the $n$-dimensional unit ball centered at $\mathbf{o}$, and let $P$ be a polytope which circumscribes $B$ and has $2 n$ facets. Is it true that

$$
\delta^{H}(B, P) \geq \sqrt{n}-1,
$$

where the equality can be realized only if $P$ is a cube?

The answer of this problem is "yes" in $R^{3}$ (see [4]). Here we present a positive solution to this problem in $R^{4}$.

Theorem 6. In four dimensions,

$$
\delta^{H}(B, P) \geq 1
$$

where the equality holds if and only if $P$ is a cube. 
To prove this theorem, we first introduce three lemmas.

Lemma 4. Let $S$ be an n-dimensional simplex of fixed volume $v$, and let $B_{r}(\mathbf{p})$ be an $n$-dimensional ball of radius $r$ and center $\mathbf{p}$. Then $S \cap B_{r}(\mathbf{p})$ has maximal volume for every $r$ if and only if $S$ is regular and $\mathbf{p}$ is the center of gravity of $S$.

Proof. Let $\mathbf{y}_{0}, \mathbf{y}_{1}, \ldots, \mathbf{y}_{n}$ be the vertices $S$ with $\mathbf{y}_{1}, \ldots, \mathbf{y}_{n}$ in the hyperplane $\left\{\left(x^{1}, x^{2}, \ldots, x^{n}\right): x^{n}=0\right\}$. Let $h>0$ be the $n$th coordinate of $\mathbf{y}_{0}$. We proceed to prove Lemma 4 by induction on $n$. Clearly, it is true for $n=1$.

It is easy to see that, for any point $\mathbf{z}_{0}$ with $z^{n}=h, \operatorname{conv}\left\{\mathbf{z}_{0}, \mathbf{y}_{1}, \ldots, \mathbf{y}_{n}\right\}$ has volume $v$. Further, any hyperplane $H_{t}=\left\{\left(x^{1}, x^{2}, \ldots, x^{n}\right): x^{n}=t\right\}, 0 \leq t \leq h$, meets $\operatorname{conv}\left\{\mathbf{z}_{0}, \mathbf{y}_{1}, \ldots, \mathbf{y}_{n}\right\}$ in a homothetic copy $((h-t) / h) \operatorname{conv}\left\{\mathbf{y}_{1}, \ldots, \mathbf{y}_{n}\right\}$ of $\operatorname{conv}\left\{\mathbf{y}_{1}, \ldots, \mathbf{y}_{n}\right\}$. By the induction assumption, these copies meet the corresponding $B_{r}(\mathbf{p}) \cap H_{t}$ in sets of maximal $(n-1)$-dimensional volume if and only if $((h-$ $t) / h) \operatorname{conv}\left\{\mathbf{y}_{1}, \ldots, \mathbf{y}_{n}\right\}$ are regular with centers lying on the vertical line of $H_{t}$ through the center of $B_{r}(\mathbf{p})$. This will be the case that $\operatorname{conv}\left\{\mathbf{y}_{1}, \ldots, \mathbf{y}_{n}\right\}$ is regular and $\mathbf{z}_{0}$ is the intersection of $H_{t}$ and the vertical line of it through $\mathbf{p}$.

Repeating this process for all the facets of $S$, Lemma 4 follows.

Lemma 5. Let $S$ be a simplex inscribed in the unit ball $B$ of $R^{n}$. Then $S \cap r B$ has maximal volume for every $r$ if and only if $S$ is regular.

Proof. Let $S=\left\{\mathbf{y}_{0}, \mathbf{y}_{1}, \ldots, \mathbf{y}_{n}\right\}$, where $\mathbf{y}_{i}=\left(y_{i}^{1}, y_{i}^{2}, \ldots, y_{i}^{n}\right)$, and assume that $y_{0}^{n}=\beta$ and $y_{i}^{n}=-\alpha$ for all $i=1,2, \ldots, n$. Then

$$
v(S \cap r B)=\int_{-\alpha}^{\beta} v^{\prime}\left(S \cap r B \cap H_{t}\right) d t,
$$

where $v^{\prime}(\cdot)$ indicates the $(n-1)$-dimensional measure and $H_{t}$ indicates the hyperplane $\left\{\left(x^{1}, x^{2}, \ldots, x^{n}\right): x^{n}=t\right\}$.

Let $\operatorname{conv}\left\{\mathbf{z}_{1}, \ldots, \mathbf{z}_{n}\right\}$ be a regular simplex lying in $H_{-\alpha}$ with center of gravity $(0, \ldots, 0,-\alpha)$ and

$$
v^{\prime}\left(\operatorname{conv}\left\{\mathbf{z}_{1}, \ldots, \mathbf{z}_{n}\right\}\right)=v^{\prime}\left(\operatorname{conv}\left\{\mathbf{y}_{1}, \ldots, \mathbf{y}_{n}\right\}\right)
$$

Take $\mathbf{z}_{0}=(0, \ldots, 0, \beta)$ and $S^{*}=\operatorname{conv}\left\{\mathbf{z}_{0}, \ldots, \mathbf{z}_{n}\right\}$. By Lemma 4 we get

$$
v(S \cap r B) \leq v\left(S^{*} \cap r B\right)
$$

where the equality holds for every $r$ if and only if $\mathbf{y}_{0}=\mathbf{z}_{0}$ and $\operatorname{conv}\left\{\mathbf{y}_{1}, \ldots, \mathbf{y}_{n}\right\}$ is regular. With minor modifications of the previous arguments Lemma 5 follows.

Lemma 6. Let $S^{\prime}$ be a spherical simplex lying within a cap $\Omega$ of radius $\rho$ of the unit ball $B$ of $R^{n}$. Then $S^{\prime}$ has maximal $(n-1)$-dimensional measure if and only if $S^{\prime}$ is regular. 
Proof. Let $S^{\prime}$ be a spherical simplex on $\partial(B)$ contained in some open hemisphere of $\partial(B)$, and let $S$ be the corresponding simplex. The affine hull of $S$ meets $\partial(B)$ in an $(n-1)$-dimensional ball with center $\mathbf{u}$ and radius $\rho, 0<\rho<1$. It is easy to see that

$$
s\left(S^{\prime}\right)=c \iint_{V(S)} e^{-|\mathbf{x}|^{2}} d x^{1} \cdots d x^{n},
$$

where $c$ is a constant depending only on $n$ and $V(S)$ indicates the cone with vertex $\mathbf{o}$ over $S$. Regarding $V(S)$ as a union of pieces $\lambda S, 0 \leq \lambda<+\infty$, then

$$
\begin{aligned}
s\left(S^{\prime}\right) & =c \int_{0}^{\infty}\left(\iint_{\lambda S} e^{-|\mathbf{x}|^{2}} d \lambda S\right) d \lambda \\
& =c \int_{0}^{\infty} \lambda^{n-1}\left(\iint_{S} e^{-\lambda^{2}|\mathbf{x}|^{2}} d S\right) d \lambda .
\end{aligned}
$$

In $S$, writing $\mathbf{x}=\mathbf{u}+\mathbf{v}$, it is easy to see that

$$
\iint_{S} e^{-\lambda^{2}|\mathbf{x}|^{2}} d S=e^{-\lambda^{2}|\mathbf{u}|^{2}} \int_{0}^{\rho} e^{-\lambda^{2} r^{2}} s_{r} d r,
$$

where $s_{r}$ indicatess the $(n-2)$-dimensional measure of $(S-\mathbf{u}) \cap \partial(r B)$, and

$$
s_{r}=\frac{d v_{r}}{d r},
$$

where $v_{r}$ denotes the $(n-1)$-dimensional measure of $(S-\mathbf{u}) \cap r B$. Then, by Lemma 5 ,

$$
\begin{aligned}
\int_{0}^{\rho} e^{-\lambda^{2} r^{2}} s_{r} d r & =\left.e^{-\lambda^{2} r^{2}} v_{r}\right|_{0} ^{\rho}+\int_{0}^{\rho} 2 \lambda^{2} r e^{-\lambda^{2} r^{2}} v_{r} d r \\
& =e^{-\lambda^{2} \rho^{2}} v_{\rho}+\int_{0}^{\rho} 2 \lambda^{2} r e^{-\lambda^{2} r^{2}} v_{r} d r \\
& \leq e^{-\lambda^{2} \rho^{2}} v_{\rho}^{*}+\int_{0}^{\rho} 2 \lambda^{2} r e^{-\lambda^{2} r^{2}} v_{r}^{*} d r
\end{aligned}
$$

where $v_{r}^{*}$ indicates the corresponding value in the regular case and the equality holds if and only if $S^{\prime}$ is regular.

By (17), (18), and (19), Lemma 6 follows.

Proof of Theorem 6. Let $P^{\prime}$ be the dual of $P$. Theorem 6 is equivalent to the following assertion: Let $P^{\prime}$ be a polytope inscribed in the four-dimensional unit ball $B$ and having eight vertices. Then

$$
\delta^{H}\left(P^{\prime}, B\right) \geq \frac{1}{2},
$$

where the equality holds if and only if $P^{\prime}$ is a regular cross polytope $W^{\prime}$.

Clearly, $\delta^{H}\left(W^{\prime}, B\right)=\frac{1}{2}$ and $\delta^{H}\left(P^{\prime}, B\right) \leq \alpha$ means

$$
(1-\alpha) B \subset P^{\prime} \text {. }
$$


As usual, we use $f_{i}$ to indicate the number of $i$-dimensional faces of $P^{\prime}$. In addition, without loss of generality, we assume that $P^{\prime}$ is simplicial. Now we proceed to prove Theorem 6 by dealing with two cases.

Case 1: $\delta^{H}\left(P^{\prime}, B\right) \leq \frac{1}{2}$ and $f_{3} \leq 16$. Projecting the facets of $P^{\prime}$ from $\mathbf{o}$ to the surface of $B$, we get $f_{3}$ spherical simplices $S_{1}^{\prime}, S_{2}^{\prime}, \ldots, S_{f_{3}}^{\prime}$. Let $s^{*}$ be the surface area of the corresponding spherical simplex determined by the facets of $W^{\prime}$. Then, by Lemma 6 ,

$$
s\left(S_{i}^{\prime}\right) \leq s^{*},
$$

where equality holds if and only if $S_{i}^{\prime}$ is deduced by a facet of a regular cross polytope. On the other hand, we have

$$
\sum_{i=1}^{f_{3}} s\left(S_{i}^{\prime}\right)=s(\partial(B)) .
$$

Therefore, $P^{\prime}$ must be a regular cross polytope.

Case 2: $\delta^{H}\left(P^{\prime}, B\right) \leq \frac{1}{2}$ and $f_{3}>16$. By the well-known Dehn-Sommerville equations (see [9]), we have

$$
\left\{\begin{aligned}
f_{0}-2 f_{1}+3 f_{2}-4 f_{3} & =-f_{0} \\
-f_{1}+3 f_{2}-6 f_{3} & =-f_{1}
\end{aligned}\right.
$$

and therefore

$$
f_{1}=f_{0}+f_{3} \geq 25 \text {. }
$$

This means that at least one vertex of $P^{\prime}$ is connected with every other vertex by an edge. In other words, in the original problem, $P$ has a facet $F$ which intersects all the other facets. Let $\mathbf{x}=F \cap B$ and assume

$$
\max _{\mathbf{y} \in F}\{\|\mathbf{o}, \mathbf{y}\|\} \leq 2,
$$

then an easy computation yields that the distance between $\mathbf{o}$ and the point of $\partial(P)$ in the opposite direction to $\mathbf{x}$ is larger than 2. In other words, we have $\delta^{H}(P, B)>1$ in this case.

As a conclusion of these two cases, Theorem 6 is proved.

Theorem 7. If $B$ is a four-dimensional ball, then

$$
b(B)=9 .
$$

Proof. It follows from Theorem 6 that

$$
b(B) \geq 9 .
$$

Write $\mathbf{e}_{1}=(1,0,0,0), \mathbf{e}_{2}=(0,1,0,0), \mathbf{e}_{3}=(0,0,1,0), \mathbf{e}_{4}=(0,0,0,1), \mathbf{e}=$ (1, 1, 1, 1),

$$
\mathbf{u}_{i}=\frac{2}{\sqrt{1+4 \varepsilon^{2}-2 \varepsilon}}\left(\mathbf{e}_{i}-\varepsilon \mathbf{e}\right),
$$


and

$$
\mathbf{v}_{i}=\frac{1}{\sqrt{1+\varepsilon+\varepsilon^{2}}}\left(-2 \mathbf{e}_{i}-\varepsilon \mathbf{e}\right) .
$$

It can be verified that, when $\varepsilon$ is small,

$$
B+\left\{\mathbf{e}, \mathbf{u}_{1}, \mathbf{u}_{2}, \mathbf{u}_{3}, \mathbf{u}_{4}, \mathbf{v}_{1}, \mathbf{v}_{2}, \mathbf{v}_{3}, \mathbf{v}_{4}\right\}
$$

blocks any other translate of $B$ from touching it. Thus,

$$
b(B) \leq 9 .
$$

Theorem 7 follows from (20) and (21).

Remark 2. Let $B$ be the unit ball in $R^{n}$, let $\Omega$ be a cap in $\partial(B)$, and let $S$ be a spherical simplex inscribed in $\Omega$. As an analogue of Lemma 1 of [2] we conjecture that $\Sigma(S) / A(S)$ attains its minimum if and only if $S$ is regular. Here $\Sigma(S)$ indicates the sum of the spherical angles of $S$ and $A(S)$ indicates the area of $S$. If this conjecture is true, by a simple mean value formula we can obtain the positive solution of Problem 4. In this way, we can give a new proof of Fejes Tóth's result in $R^{3}$.

\section{Acknowledgment}

We are very grateful to the referee for his helpful comments and remarks.

\section{References}

1. K. Ball, Ellipsoids of maximal volume in convex bodies, Geom. Dedicata 41 (1992), 241-250.

2. H.S.M. Coxeter, L. Few, and C.A. Rogers, Covering space with equal spheres, Mathematika 6 (1959), $147-157$.

3. A. Dvoretzky, Some results on convex bodies and Banach spaces, Proc. Symp. on Linear Spaces, Jerusalem, 1961, pp. 123-160.

4. L. Fejes Tóth, Lagerungen in der Ebene, auf der Kugel und in Raum, Springer-Verlag, Berlin, 1972.

5. P.M. Gruber and C.G. Lekkerkerker, Geometry of Numbers, North-Holland, Amsterdam, 1987.

6. B. Grünbaum, On a conjecture of Hadwiger, Pacific J. Math. 11 (1961), 215-219.

7. B. Grünbaum, Measures of symmetry for convex sets, Convexity, Proc. Sympos. Pure Math., Vol. 7, American Mathematics Society, Providence, RI, 1963, pp. 233-270.

8. F. John, Extremum problems with inequalities as subsidiary conditions, Courant Ann. Volume, Interscience, New York, 1948, pp. 187-204.

9. K. Leichtweiß, Konvexe Mengen, Springer-Verlag, Berlin, 1980.

10. P. McMullen and G.C. Shephard, Convex Polytopes and the Upper Bound Conjecture, Cambridge University Press, Cambridge, 1971.

11. J. Steiner, Einfache Beweise der isoperimetrischen Hauptsätze, J. Reine Angew. Math. 18 (1838), 281-296.

12. C. Zong, Packing and Covering, Ph.D. Thesis, TU Wien, 1993.

13. C. Zong, Some remarks concerning kissing numbers, blocking numbers and covering numbers, Period. Math. Hungar. 30 (1995), 233-238.

14. C. Zong, Strange Phenomena in Convex and Discrete Geometry, Springer-Verlag, New York, 1996.

Received December 11, 1998, and in revised form October 5, 1999. Online publication May 3, 2000. 